\title{
Traitement des dysfonctions érectiles par auto-injections intracaverneuses : comparaison des résultats du moxisylyte et de la prostaglandine E1
}

\author{
J. Buvat, A. Lemaire, M. Herbaut-Buvat \\ Association pour l'étude de la pathologie de l'appareil reproducteur et la psychosomatique, \\ (EPARP), 49 rue de la Bassée, 59000 Lille, France
}

Version française légèrement modifiée de : J. Buvat, A. Lemaire, $M$. Herbaut-Buvat : Intracavernous pharmacotherapy : comparison of Moxisylyte and Prostaglandin E1. International Joumal of Impotence Research, 1996, 8, 41-46.

\section{RESUME}

Nous rapportons dans cette étude les résultats d'auto-injections intracaverneuses des 2 premières substances vaso-actives proposées pour réduire les taux relativement élevés de priapisme et de fibrose observés après Papavérine, soit le Moxisylyte (Mox.) et la Prostaglandine E1 (PGE1). Chaque substance fut utilisée chez 130 patients avec des durées moyennes de suivi comparables (14.8 mois avec le Mox. et 14.6 avec la PGE1). La PGE1 s'avéra significativement plus efficace (bon résultat chez $71 \%$ des sujets contre 50 $\%$ avec le Mox.), spécialement chez les sujets artériels (respectivement $96 \%$ vs $46 \%$ ). En contre partie, elle induisit des érections prolongées chez un plus grand nombre de sujets (11 versus 1 avec le Mox.), parmi lesquels 2 priapismes, et induisit plus souvent une douleur (12 sujets contre 1 avec le Mox.). La survenue de plaques ou de nodules fibreux fut rare (respectivement 2 et 3 sujets). Malgré sa meilleure tolérance, le taux de continuation du Mox. fut significativement plus faible que celui de la PGE1. La PGE1 pourrait être la substance de premier choix dans la plupart des cas. Le Mox. pourrait être utile pour les médecins peu expérimentés, ainsi que chez les sujets hypersensibles aux injections, et chez ceux qui ressentent une douleur significative après PGE1.

Mots clés : Dysfonction érectile, traitement, auto-injections, Moxisylyte, Prostaglandine E1.

La découverte par Virag et Brindley de la possibilité d'induire une érection par injection intracaverneuse de substance vasoactive a révolutionné le diagnostic et le traitement des dysfonctions érectiles (DE) [1, $2]$. Cependant, les 2 premières substances utilisées, la papavérine et la phénoxybenzamine, s'avérèrent exposer à des risques relativement élevés de priapisme et de fibrose des corps caverneux, susceptibles d'aboutir à une impuissance définitive rebelle à tous les types de traitement $[3,6]$. Ceci rendit souhaitable l'emploi de substances mieux tolérées.

Plusieurs substances de deuxième génération ont été proposées dans cette perspective. Les deux premières disponibles dans notre centre furent l'alpha-bloqueur Moxisylyte (Mox.), ou thymoxamine, dont nous avons développé en France l'utilisation intracaverneuse à partir de $1985[4,7]$, et la Prostaglandine E1 (PGE1) qui fut d'abord utilisée par Ishii en 1986 [8] et que nous avons nous-même commencé d'employer en 1989. Nous avons rapporté précédemment que le Moxisylyte donnait des taux beaucoup plus faibles d'érections prolongées et de nodule fibreux que la papavérine [9]. Dans une étude avec comparaison intra- 
individuelle, Porst [10] démontra également une diminution du risque de priapisme après PGE1 comparée à la papavérine ou à l'association papavérine-phentolamine. Dans cette étude rétrospective, nous comparons les résultats obtenus avec le Mox. et la PGE1 chez des sujets traités par autoinjections.

\section{SUJETS ET METHODES}

Les sujets de cette étude furent d'une part les 130 premiers impuissants inclus dans la phase initiale (recherche de la dose minima efficace et apprentissage des injections) de notre programme de traitement par autoinjections de Mox. (Juin 1985 à Décembre 1993), et des 130 premiers inclus dans la phase initiale de notre programme d'autoinjections de PGE 1 (Octobre 1989 à Décembre 1993). Ils avaient de 20 à 71 ans (moyenne 49.2 \pm 9.1 dans le groupe Mox., et $52.7 \pm 10.4$ dans le groupe PGE1, différence non significative : NS).

Les caractéristiques du Moxisylyte ont été rapportées précédemment $[7,9,12]$. Il se présente en flacons de 10 ou $20 \mathrm{mg}$ à dissoudre juste avant l'injection. Jusque 1990, nous disposions également d'une présentation à $30 \mathrm{mg}$, qui n'a pas été mise sur le marché. En ce qui concerne la PGE1 (alprostadil), nous avons utilisé 3 présentations différentes : une préparation magistrale chez 85 sujets. Il s'agit d'ampoules de $1 \mathrm{ml}$ contenant $500 \mu \mathrm{g}$ de PGE1 en solution alcoolique, que nous avons diluées dans 49 $\mathrm{ml}$ de sérum salé de façon à obtenir une solution à $10 \mu \mathrm{g}$ par ml. L'Alprostadil commercialisé en 1994 fut utilisé chez 25 sujets. Il se présente en flacons contenant $20 \mu \mathrm{g}$ de PGE1 lyophilisée à dissoudre dans son solvant juste avant l'injection. Enfin, 20 impuissants ont été traités par l'Alprostadil-Alphadex, également en ampoules de 20 $\mu$ g lyophylisat à dissoudre juste avant l'injection. Les deux derniers sous-groupes participaient à 2 grands essais multicen- triques européens, conduits en accord avec les bonnes pratiques cliniques, et commencés respectivement en 1993 et 1991. La répartition des substances et des présentations ne fut pas randomisée, mais basée sur leur disponibilité, et, lorsque les deux substances étaient disponibles simultanément, sur le résultat de tests préliminaires au cabinet.

Les impuissants ont été répartis en différentes catégories étiologiques en fonction des explorations usuellement pratiquées dans notre centre, à savoir interrogatoire, examen clinique, évaluation psychologique du sujet et si possible de sa partenaire, dosages hormonaux (testostérone, prolactine), étude Doppler des artères péniennes, couplée depuis fin 1989 à une injection intracaverneuse de $10 \mu \mathrm{g}$ de PGE1 (pharmacodoppler pulsé), et si nécessaire pléthysmographie nocturne avec rigidimétrie (Rigiscan) couplée à des enregistrements de sommeil complets, cavernosométrie et cavernosographie, mesure de la latence du réflexe bulbo-caverneux et enregistrement des potentiels évoqués somesthésiques du nerf honteux interne. Trois groupes ont ainsi été constitués : prépondérance psychogène, prépondérance organique, ou mixte. Les cas organiques ont été ensuite séparés en artériels, veineux (dysfonction veinoocclusive), neurologique et non déterminée.

Les auto-injections ont été proposées parmi plusieurs options, non seulement aux impuissants apparemment principalement organiques, mais également à certains cas principalement psychogènes ou mixtes, après échec d'une psychothérapie ou d'une sexothérapie, ou lorsque ces traitements n'étaient pas adaptés aux patients ou couples correspondants. Dans ces cas, un soutien psychologique et un conseil sexuel furent poursuivis pendant l'apprentissage et la surveillance du traitement.

Les sujets ont d'abord été testés avec $10 \mathrm{mg}$ de Moxisylyte, ou $10 \mu \mathrm{g}$ de PGE1. La dose 
initiale a été réduite à $5 \mathrm{mg}$ ou $5 \mu \mathrm{g}$ dans les cas neurologiques purs. Dans la mesure où le résultat de l'injection peut être meilleur à domicile, dans l'intimité, que dans un environnement médical [7], nous n'avons pas exigé que les injections tests obtiennent une rigidité dans notre centre pour inclure le sujet dans le programme thérapeutique, particulièrement si celui-ci nous semblait stressé ou gêné par le procédé. L'apprentissage fut fait en 2 à 4 sessions selon l'habileté du sujet, y compris l'exécution complète d'une injection en face de nous. Si nécessaire, la dose initiale fut augmentée après les premiers essais à domicile. Nous avons utilisé des aiguilles $27 \mathrm{G}$ jusque 1989 , puis des aiguilles 30 G1/2. Des consultations de contrôle ont eu lieu après 2 à 4 semaines, puis tous les 2 à 4 mois selon la fréquence des injections. Elles ont comporté une évaluation des résultats et des effets indésirables, et un examen soigneux du pénis.

\section{RESULTATS}

Le groupe Mox. comprenait un peu plus de cas psychogènes ( 69 versus 53 ) et moins de cas principalement organiques (43 versus 52) (NS).

Les 130 impuissants Mox. ont été suivis 1931 mois (moyenne 14.8). Ils ont fait 6090 injections (47 par sujet, 3.2 par mois). Le suivi le plus long a été de 84 mois, avec 705 injections. Les chiffres correspondants pour la PGE1 ont été 1902 mois (moyenne 14.6) et 7480 injections ( 58 par sujet, 4 par mois, soit $20 \%$ de plus que dans le groupe Mox.). Trois impuissants ont utilisé la PGE1 pendant 42 mois, et l'un d'eux a fait 390 injections.

L'efficacité a été qualifiée de bonne quand plus de $75 \%$ des injections étaient suivies de rapports satisfaisants, et de moyenne lorsque le sujet était amélioré dans une proportion moindre des rapports, ou que sa rigidité était incomplète ou trop brève. Nous avons considéré guéris les sujets qui avaient retrouvé des érections spontanées et étaient capables de rapports normaux plus de 3 mois après avoir stoppé les injections. Nous avons parlé de sujet amélioré lorsqu'il pouvait avoir des rapports satisfaisants sans injection au moins 2 fois plus souvent qu'avant de commencer le traitement par auto-injections, bien qu'il doive continuer d'utiliser les injections de façon intermittente. L'efficacité d'ensemble est détaillée dans le tableau 1. La PGE1 s'est avérée significativement plus efficace (Chi 2 : 11.97, $\mathrm{p}<0.01$ ). Cependant les proportions des sujets guéris et améliorés furent identiques dans les deux groupes.

Lorsque nous avons examiné les résultats en fonction de l'étiologie, la supériorité de la PGE1 n'apparut plus significative que dans le groupe artériel (Tableau 2). La différence d'efficacité fut plus faible dans le groupe principalement psychogène et surtout chez les neurologiques purs. Le Mox.

Tableau 1 : Efficacité chez l'ensemble des sujets.

\begin{tabular}{|c|c|c|c|c|c|}
\hline $\begin{array}{l}\text { Substance } \\
\text { (nb de cas) }\end{array}$ & Bonne & Moyenne & $\begin{array}{l}\text { Médiocre } \\
\text { ou nulle }\end{array}$ & $\frac{\text { Réapp. d'ére }}{\text { Guérison }}$ & $\frac{\text { ons spontanées }}{\text { Amélioration }}$ \\
\hline $\begin{array}{l}\text { Moxisylyte } \\
(\mathrm{n}=130)\end{array}$ & $\begin{array}{c}65 \\
50 \%\end{array}$ & $\begin{array}{c}24 \\
18 \%\end{array}$ & $\begin{array}{c}41 \\
32 \%\end{array}$ & $\begin{array}{c}15 \\
11,5 \%\end{array}$ & $\begin{array}{c}16 \\
12,3 \%\end{array}$ \\
\hline $\begin{array}{l}\text { PGE1 } \\
(n=130)\end{array}$ & $\begin{array}{c}92 \\
71 \%\end{array}$ & $\begin{array}{c}16 \\
12 \%\end{array}$ & $\begin{array}{c}22 \\
17 \%\end{array}$ & $\begin{array}{c}12 \\
9,2 \%\end{array}$ & $\begin{array}{c}13 \\
10 \%\end{array}$ \\
\hline
\end{tabular}


s'avéra inefficace dans tous les cas de dysfonction veino-occlusive, tandis que la PGE1 fut pleinement efficace chez $31 \%$ des 13 cas de ce type.

Chez 3 sujets, ( 2 neurogènes et 1 psychogène), des doses inférieures à $2 \mu \mathrm{g}$ de de PGE1 furent efficaces pendant plus d'un an. Les valeurs moyennes et extrêmes des doses efficaces dans chaque sous-groupe étiologique sont précisées dans le tableau 3. Chez les vasculaires, les doses efficaces de PGE1 sont clairement plus élevées que chez les psychogènes ( $<5 \mu$ g chez 3 sur 12 ). Ce sont les sujets avec dysfonction veino-occlusive qui ont nécessité les doses les plus élevées ( $>20 \mu \mathrm{g}$ chez $6 / 12$ contre $0 / 6$ sujets artériels). Ces différences sont moins nettes avec le Mox., qui n'obtint aucun résultat dans les dysfonctions veino-occlusives et chez plus de $50 \%$ des artériels.
Les effets indésirables sont détaillés dans le tableau 4. Le nombre des sujets ayant présenté des érections durant plus de 2 et 3 heures s'avéra significativement plus élevé dans le groupe PGE1 (une seule érection prolongée dans le groupe Mox., s'étant spontanément interrompue après $4 \mathrm{~h}$ ). Cependant, 3 sujets seulement eurent des érections de plus de $4 \mathrm{~h}$ avec la PGE1 (interruption spontanée chez 2 , après 4 et 7 heures, dérivation chirurgicale dans le dernier cas, après $36 \mathrm{~h}$, sans aucune séquelle. Malgré nos recommandations, ce sujet s'était fait 3 injections le même jour, et ne nous appela qu'après $33 \mathrm{~h}$ d'érection prolongée, laquelle ne céda pas après injection intracaverneuse de métaraminol). Les érections prolongées survinrent presque exclusivement chez les sujets neurogènes et psychogènes.

Tableau 2 : Proportion des sujets avec bonne efficacité des injections en fonction de la cause prépondérante de la $\mathrm{DE}$ (nombre de cas avec bonne efficacité/nb total des cas traités).

\begin{tabular}{lcccc}
\hline Substance & psychogènes & artériels & neurogènes & veineux \\
\hline Moxisylyte & $39 / 69$ & $11 / 24$ & $10 / 14$ & $0 / 5$ \\
& $56 \%$ & $46 \%$ & $71 \%$ & $0 \%$ \\
PGE1 & $42 / 53$ & $26 / 27$ & $10 / 12$ & $4 / 13$ \\
& $79 \%$ & $96 \%$ & $83 \%$ & $31 \%$ \\
Khi 2 & 5,9 & 13,8 & 0,6 & - \\
p & NS & $<0,001$ & NS & \\
\hline
\end{tabular}

Tableau 3 : Doses efficaces (extrêmes et moyennes + écart type) selon l'étiologie de la dysfonction érectile.

\begin{tabular}{lcccc}
\hline Substance & Psychogènes & Artériels & Neurogènes & Veineux \\
\cline { 1 - 3 } Moxisylyte & 5 à 30 & 10 à 30 & 2,5 à 30 & \\
$(\mathrm{mg})$ & $15,7 \pm 7,9$ & $20,8 \pm 8,6$ & $18,2 \pm 9,8$ & - \\
PGE1 & 1,4 à 40 & 2 à 20 & 1 à 40 & $15-60$ \\
$(\mu \mathrm{g})$ & $10,6 \pm 7$ & $15,4 \pm 5,4$ & $8,9 \pm 5,4$ & $23,7 \pm 12$ \\
\hline
\end{tabular}


En ce qui concerne la douleur, nous n'avons pas pris en compte la simple tension pénienne souvent rapportée comme douleur dans la littérature, bien qu'elle ne soit pas réellement ressentie comme douloureuse par le sujet, mais uniquement les sensations réellement déplaisantes et douloureuses. Cet effet indésirable a également été rapporté par une proportion plus élevée des sujets sous PGE1 (9.2\%). Dans 6 cas, la douleur fut purement locale (au site de l'injection), constante dans 2 seulement de ces cas, et concernant moins de $50 \%$ des injections dans les 4 autres. Dans les 6 autres cas, elle était diffuse, et persistait jusqu'à la fin de l'érection. Son intensité ne fut majeure qu'une fois, chez le seul sujet ayant arrêté le traitement pour cet effet indésirable. Elle était discrète ou modérée dans les autres cas. Elle diminua lorsqu'il fut possible de réduire la dose de PGE1, ou, dans plusieurs cas, avec le temps sans même réduire la dose. Un seul sujet du groupe Mox. rapporta une douleur significative.
Des plaques ou des nodules fibreux apparurent sous traitement chez 3 sujets du groupe PGE1 et 2 du groupe Moxisylyte. Ceci ne fut jamais suivi d'une diminution de la réponse érectile ou une angulation pénienne. Nous avons alors conseillé de réduire de $50 \%$ la fréquence des injections, et de les faire à distance de l'anomalie fibreuse. Dans ces conditions ces lésions n'ont pas augmenté malgré la poursuite des injections. Elles disparurent en un an chez un sujet de chaque groupe.

Un échappement aux injections, défini par la nécessité d'en augmenter la dose d'au moins $100 \%$ du fait d'une diminution progressive de la réponse, survint avec la même fréquence dans les 2 groupes (14 et $13 \%$ ). Quelques autres effets indésirables mineurs survinrent exclusivement dans le groupe Mox. (Tableau 4).

Nous avons calculé le taux de poursuite des 2 traitements à intervalles de 6 mois (Figure 1). Pour chaque date anniversaire $(6,12$, 18,24 mois etc), nous avons compté les

Tableau 4 : Effets indésirables (nombres de sujets concernés).

\begin{tabular}{|c|c|c|}
\hline Effet indésirable & Moxisylyte & PGE1 \\
\hline Erection $>2 \mathrm{~h}$ & $1(0,7 \%)$ & $11(8,4 \%)^{* *}$ \\
\hline Erection $>3 \mathrm{~h}$ & $1(0,7 \%)$ & $9(6,9 \%)$ \\
\hline Erection $>4 \mathrm{~h}$ & $1(0,7 \%)$ & $3(2,2 \%)$ \\
\hline Erection $>6 \mathrm{~h}$ & - & $2(1,5 \%)$ \\
\hline $\begin{array}{l}\text { Parmi lesquelles ayant } \\
\text { nécessité un traitement }\end{array}$ & - & $1(0,7 \%)$ \\
\hline Douleur locale & $1(0,7 \%)$ & $6(4,6 \%)$ \\
\hline Douleur diffuse & - & $6(4,6 \%)$ \\
\hline Fibrose 1 & $2(1,5 \%)$ & $3(2,3 \%)$ \\
\hline Echappement & $18(14 \%)$ & $17(13 \%)$ \\
\hline Ejaculation retardée & $5(3,8 \%)$ & $1(0,7 \%)$ \\
\hline Lipothymie / vertige 2 & $2(1,5 \%)$ & - \\
\hline Somnolence 2 & $1(0,7 \%)$ & - \\
\hline Tachycardie $^{2}$ & $1(07 \%)$ & - \\
\hline Rhinorrhée ${ }^{2}$ & $1(0,7 \%)$ & - \\
\hline
\end{tabular}

${ }^{1}$ : plaques ou nodules $;^{2}$ : corrélé chronologiquement aux injections; ${ }^{* *}$ khi $211,97, p<0,01$ 
sujets ayant poursuivi le traitement pendant au moins le nombre de mois correspondants, et nous avons divisé leur nombre par le nombre total des patients qui auraient encore été en traitement après ce nombre de mois si aucun n'avait interrompu les injections (taux de poursuite d'ensemble). Dans ce calcul, nous avons considéré que les perdus de vue avaient interrompu le traitement, dans la mesure où, dans notre région, la PGE1 n'était disponible dans aucun autre centre ou cabinet médical jusqu'à la fin de l'étude, tandis que le Mox. ne fut disponible en dehors de notre centre que pendant la dernière année (1993). Dans la mesure où il était logique que les patients ne continuent pas le traitement en cas d'échec immédiat, ou de récupération d'érections spontanées, nous avons calculé un second taux de poursuite après exclusion des échecs précoces (moins de 3 mois d'auto-injections) et des patients guéris, par conséquent limité aux seuls patients qui auraient du continuer le traitement s'il leur avait procuré initialement de bonnes érections.

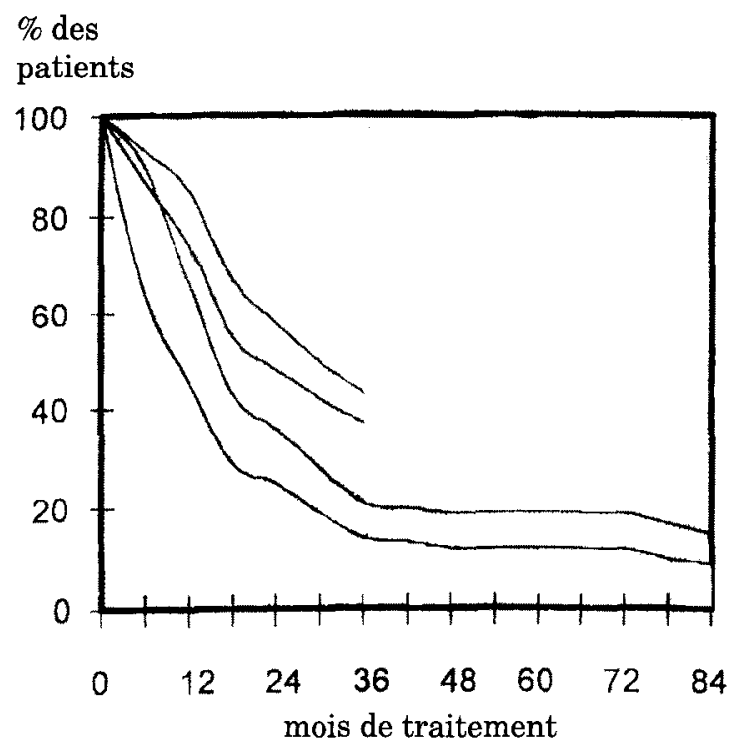

Figure 1 : Taux de poursuite des autoinjections de Moxisylyte et PGE1 dans l'ensemble des cas, et après exclusion des échecs précoces et des guérisons.
Les deux taux de poursuite étaient significativement plus élevés dans le groupe PGE1 (taux d'ensemble : $87 \%$ vs $69 \%$ dans le groupe Mox. à 6 mois ; $74 \%$ vs $46 \%$ à 12 mois, $55 \%$ vs $29 \%$ à 18 mois $(\mathrm{p}<0.001$ pour ces 3 taux), $48 \%$ vs $25 \%$ à 24 mois (p $<0.01$ ) et $37 \%$ vs $14 \%$ à 36 mois ( $<<$ $0.05)$ ). Après exclusion, $85 \%$ vs $66 \%$ à 12 mois ( $\mathrm{p}<0.01), 58 \%$ vs $36 \%$ à 24 mois ( $\mathrm{p}<$ $0.05), 43 \%$ vs $25 \%$ à 36 mois (NS). Douze pour cent des sujets ayant commencé les injections de Mox. étaient encore en traitement après 4,5 et 6 années, et $9 \%(15 \%$ des cas après exclusion des échecs précoces et des guérisons) après 7 ans.

\section{DISCUSSION}

Notre étude confirme comme de nombreuses autres $[3,4,14]$ l'efficacité du traitement par auto-injections chez une proportion importante des impuissants (résultats bons ou acceptables chez $83 \%$ des sujets du groupe PGE1 et $68 \%$ de ceux du groupe Mox.). Ce traitement est plus efficace chez les sujets à corps caverneux et vaisseaux intacts (neurogènes et psychogènes), qui nécessitent des doses plus faibles. En revanche, ces sujets ont des risques plus importants d'érection prolongée et de priapisme. C'est là une raison supplémentaire pour ne pas considérer les injections intracaverneuses comme un traitement de première intention de l'impuissance psychogène. Une approche psychologique, comportant au moins un conseil sexuel élaboré, doit être envisagée en première intention.

Bien que des réserves doivent être émises dans l'interprétation de nos résultats, dans la mesure où notre étude n'était ni prospective, ni randomisée, ils permettent d'esquisser les principales caractéristiques des 2 substances utilisées. La PGE1 semble plus efficace, l'avantage étant significatif chez l'ensemble des sujets et chez les artériels. Elle s'est d'ailleurs avérée supérieure à la papavérine dans plusieurs études avec com- 
paraison intra-individuelle $[10,15,16]$, alors que nous avons montré que le Mox. était beaucoup moins puissant que la papavérine [7].

En contrepartie, du fait de sa moindre puissance le Mox. expose nettement moins aux érections prolongées et au priapisme. Nous avions trouvé précédemment un taux d'érections prolongées de plus de 4 heures de seulement $1 \%$ chez les 170 premiers sujets traités par Mox. dans notre centre, contre $14 \%$ de ceux testés par la papavérine [7] ; en ce qui concerne les auto-injections, nos taux d'érections prolongées étaient de $1.3 \%$ avec le Mox. contre $8.8 \%$ avec la papavérine [9]. Mais bien que rare, cet effet indésirable peut également survenir avec le Mox. chez les sujets hypersensibles ( 3 des 12 paraplégiques testés par Costa et coll [12]) et 2 priapismes ont d'ailleurs été rapportés avec cette substance $[7,14]$.

Les risques auxquels exposent les érections prolongées induites par la PGE1 semblent cependant modestes. Au contraire de ce qu'on a rapporté avec la papavérine [3, 4], toutes les érections prolongées de cette étude ont disparu spontanément, à l'exception de celle qui survint chez un patient ayant fait 3 injections le même jour. Aucune ne fut suivie d'une détérioration de la capacité érectile. De plus le taux des érections prolongées de plus de 4 heures est clairement plus faible que les 8.7 à $9.5 \%$ rapportés pour la papavérine ou l'association papavérine-phentolamine dans les revues de la littérature $[3,4]$. Trois études avec comparaison intra-individuelle $[10,15$, 16] ont d'ailleurs confirmé que ce risque était significativement plus faible avec la PGE1 qu'avec la papavérine (1.4\% vs $4.3 \%$ de l'ensemble des sujets, $\mathrm{p}<0.05$ ) et l'association papavérine-phentolamine $(6 \%, \mathrm{p}<$ 0.01) [14].

Que la PGE1, et plus encore le Mox., induisent significativement moins de priapismes que la papavérine, n'implique pas qu'on puisse abandonner les précautions en usage pour les premières injections depuis l'emploi de la papavérine, puisqu'on a observé des priapismes avec les 2 substances. Il faut particulièrement se limiter à des doses faibles pour la première injection $(10 \mathrm{mg}$ de Mox ou $10 \mu$ g de PGE1, ou même 5 mg ou 5 $\mu \mathrm{g}$ chez les sujets jeunes, suspects d'être exclusivement psychogènes ou neurogènes, puisque notre étude confirme que la grande majorité des érections prolongées et des priapismes provient de ces catégories).

On associe classiquement l'effet indésirable douleur à l'injection de PGE1 [3, 4]. Une revue de la littérature fixait sa fréquence à $23 \%$ en ce qui concerne la première injection [14]. La douleur diminue souvent ultérieurement si on réduit la dose, ou même sans la modifier $[14,17,18]$. Ceci explique que cet effet indésirable soit moins souvent rapporté dans les traitements par autoinjections. Son importance est souvent surestimée. Les études avec comparaison intra-individuelle ont montré que les phénomènes douloureux sont plus fréquents avec la papavérine [15]. Parmi les principaux types de douleurs, seule la douleur diffuse est plus fréquente avec la PGE1 [16]. Très peu de sujets interrompent la PGE1 pour ce motif (1/130 dans notre étude, 2/162 dans l'étude prospective de Porst [18], et 2/144 dans l'étude prospective de Giuliano [17]). Cependant la douleur est nettement plus rare avec le Mox. [7, 18], et dans ce cas exclusivement locale.

Les taux de lésions fibreuses ont été plus faibles dans les 2 groupes que les $5.4 \%$ calculés après compilation des études rétrospectives consacrées à la papavérine et à l'association papavérine-phentolamine [3]. Des taux beaucoup plus élevés ont été rapportés dans 2 études prospectives ( $31 \%$ et $57 \%$ après un an $[5,6])$. De plus, il n'est survenu dans notre étude ni angulation pénienne, ni diminution de la réponse érectile chez les sujets ayant installé une fibrose, alors qu'avec la papavérine plusieurs 
sujets perdirent toute réponse aux injections après l'apparition de tels nodules fibreux [3]. Deux études prospectives récentes ont trouvé des taux de lésions fibreuses plus élevés avec la PGE1 : $3 \%$ à 6 mois [17], incluant une angulation pénienne, et $9.3 \%$ à 24 mois [18], incluant un cas de diminution de la réponse érectile et un avec angulation. Dans les deux études, aucune lésion n'était apparue avant 6 mois. Dans la seconde [18], la fibrose était corrélée avec des signes indirects de mauvaise technique d'injection. Ces données suggèrent que, bien que moins fréquentes qu'avec la papavérine, les complications fibreuses peuvent survenir avec la PGE1 et le Mox., ce qui rend nécessaire une prévention sous la forme d'un apprentissage soigneux de la technique d'injection.

Bien que le Mox. soit mieux toléré que la PGE1, son taux d'abandon fut significativement plus élevé, le situant dans la zone des 32 à $66 \%$ rapportés avec la papavérinephentolamine $[5,6,19,20]$, et suggérant qu'il satisfait peut-être moins les patients que la PGE1.

En conclusion, nos résultats montrent que la PGE1 pourrait être la substance de premier choix pour les injections intracaverneuses chez la plupart des patients, à condition d'être employée par des médecins expérimentés dans ce domaine, et de limiter la dose à $5 \mu \mathrm{g}$ chez les sujets suspects d'être neurogènes ou psychogènes. Le Mox. pourrait être préférable dans certains cas : médecins peu expérimentés, sujets avec hypersensibilité aux injections, et douleur significative après PGE1, ne diminuant pas en dépit de la répétition des injections, éventuellement à dose plus faible.

Remerciements : Mlle Nathalie BARLET, pour son aide secrétariale.

\section{REFERENCES}

1. VIRAG R. Intracavernous injection of papavérine for erectile failure.Lancet 1982; $2: 938$.
2. BRINDLEY GS. Cavernosal alpha-blockage : a new technique for investigating and treating erectile impotence. Br J Psychiatry 1983; 143 : 332-337.

3. Junemann KP, ALken P. Pharmacotherapy of erectile dysfunction : a review. Int. J. Impotence Res. 1989; $1: 71-93$.

4. Buvat J, Buvat-Herbaut M, Lematre A, Marcolin G. Applications diagnostiques et thérapeutiques des injections intracaverneuses de drogues vasoactives dans l'impuissance. Plaidoyer pour l'utilisation des drogues facilitatrices. J Urol. 1989; 95 : 33-39.

5. Levine SB, Althof SE, Turner LA, Risen CB, BoDNER DR, KURSCH ED, RESNICK MI. Side effects of self administration of intracavernous papavérine and phentolamine for the treatment of impotence. J Urol. 1989; 141 : 54-57.

6. Lakin M, Montague D, Medendorp SV, Tesar L; SCHOVER LR. Intracavernous injection therapy : analysis of results and complications. $J$ Urol. 1990; $143: 1138-1141$.

7. Buvat J, Lemaire A, Buvat-Herbaut M, Marcolin G. Safety of intracavernous injections using an alphablocking agent. J Urol. 1989; 141 : 1364-1367.

8. Ishi N, Watanabe H, IrISAwa C. Therapeutic trial with prostaglandin $\mathrm{E} 1$ for organic impotence. Jap. J Urol. 1986; 77 : 954-962.

9. Buvat J, Buvat-Herbaut M, Lemaire A, Marcolin G. Reduced rate of fibrotic nodules in the cavernous bodies folowing auto-intracavernous injection of moxisylyte compared to papavérine. Int J Impotence Res. 1991; $3: 123-128$.

10. Porst H, Van Ahlen H. Pharmakon-induzierte Priapismen. Ein Erfahrungsbericht über 101 Fälle. Urologe A. 1989; 28 : 84-87.

12. Costa P, Sarrazin B, Bressolle F, Colson MH, Bondil P, SAUDUBRAY F. Efficiency and side effects of intracavernous injections of moxisylyte in impotent patients : a dose-finding study versus placebo. J Urol. 1993; 149 : 301-305.

13. Fraccaro a, Grion aM, Gaion RM, Ricatti MG, Pescatori ES, Calabro A. Prostaglandin E1 diluted solution : preparation technique and stability evaluation. Int J Impotence Res. 1993; 5 : 43-45.

14. Buvat J, Buvat-Herbaut M, Lemaire A, Marcolin G. Les injections intracaverneuses de Prostaglandine E1 : un réel progrès dans l'efficacité et la sécurité des injections intracaverneuses. Andrologie. 1991; $1: 78-92$.

15. Chiang HS, Wen TC, Wu CC, Chiang WH. Prostaglandin $\mathrm{E} 1$ versus papavérine for diagnosis of erectile dysfunction. Int J Impotence Res. 1990; 2 : 127-130. 
16. Wetterauer U. Intra-individual comparison of papaverine-phentolamine combination versus prostaglandin E1 in intracavernous injection therapy for erectile dysfunction. Int $J$ Impotence Res. $1990 ; 2$ : 238-239.

17. Giuliano F, Blanchet P, Banzet O, Lancrenon $S$, Benoit G, Jardin A et le GFEA. Troubles de l'érection : efficacité et tolérance à 6 mois des autoinjections intracaverneuses d'Alprostadil. Presse Med. 1994; 23 : 1516-1521.

18. Porst H, Buvat J, Hauri D, Krotovsky GS, MeuLeman EJH, Michal V, Wagner G, Wespes E. Self injection therapy with prostaglandin E1.Long term results of an international multicenter study according to the GCP-standard. Int J Impotence Res. 1994; 6, suppt 1, D108.

19. Sidi AA, Cameron JS, Duffy LM, Lange PH. Intracavernous drug-induced erections in the management of male erectile dysfunction : experience with 100 patients. J Urol. 1986; 135 : 704706.

20. Van dRIEL MF, Mooibroek JJ, Van de Wiel HBM, MENSINK HJA. Intracavernous pharmacotherapy : psychological, sexological, and medical aspects. Int J Impotence Res. 1991; 3 : 95-104.

\section{ABSTRACT \\ Intracavernous pharmacotherapy : comparison of Moxisylyte and Prostaglandin E1}

J. Buvat, A. Lemaire, M. Herbaut-Buvat

We report in this retrospective study the results obtained with the 2 first drugs proposed to reduce the relatively high rates of priapism and fibrosis bound to the papaverine intracavernous injections, ie the alpha-blocking agent Moxisylyte (Mox.), and prostaglandin E1 (PGE1). Each drug was used for auto-injections in 130 patients with a comparable mean follow up (14.8 months with Mox. compared to 14.6 with PGE1). PGE1 proved to be significantly more efficacious (good results in $71 \%$ of the patients versus $50 \%$ with Mox.), especially in the arteriogenic patients (respectively $96 \%$ vs 46\%). Conversely PGE1 induced prolonged erections in significantly more patients (11 vs 1 with Mox.), including 2 priapisms, and also induced pain in more patients (12 vs 1 with Mox.). The rate of fibrotic nodules and plaques was low (2 and 3 patients). Despite the better tolerance of Mox., its continuation rate was significantly lower than that of PGE1. PGE1 can be the first choice agent in most cases. Mox. is mainly indicated in the patients with supersensitivity to the injections and in those with significant pain following PGE1.

Key words : Erectile dysfunction, treatment, autoinjections, Moxisylyte, prostaglandin E1. 